\title{
High-performance Cardiovascular Medicine: Artificial Intelligence for Coronary Artery Disease
}

H.M.K.K.M.B. Herath ( $\square$ kasunherathlive@gmail.com )

Open University of Sri Lanka https://orcid.org/0000-0002-1873-768X

G.M.K.B. Karunasena

Open University of Sri Lanka

H.D.N.S. Priyankara

Open University of Sri Lanka

B.G.D.A. Madhusanka

Open University of Sri Lanka

\section{Research Article}

Keywords: Cardiovascular diseases, Coronary Artery Disease, Disease Prediction, Machine Learning, Smart Medicine

Posted Date: June 22nd, 2021

DOI: https://doi.org/10.21203/rs.3.rs-642228/v1

License: (c) (1) This work is licensed under a Creative Commons Attribution 4.0 International License. Read Full License 


\title{
High-performance Cardiovascular Medicine: Artificial Intelligence for Coronary Artery Disease
}

\author{
H.M.K.K.M.B. Herath ${ }^{1 *}$, G.M.K.B. Karunasena ${ }^{2}$, H.D.N.S. Priyankara ${ }^{3}$, \\ B.G.D.A. Madhusanka ${ }^{4}$ \\ 1, 2, 3, 4 Faculty of Engineering Technology, The Open University of Sri Lanka \\ * Corresponding Author: kasunherathlive@gmail.com
}

\begin{abstract}
Cardiovascular disease (CVD) is identified as the leading cause of death globally, according to the World Health Organization (WHO). Approximately 17.9 million people are dying due to cardiovascular disease, which is an estimation of $31 \%$ of all deaths worldwide. CVDs are generally affecting the heart and blood vessels in the human body. Since healthcare is an essential factor for a country and its economy, researchers are looking for solutions to predict disease before getting into serious problems. This research introduces a method to development of an algorithm to predict coronary artery disease based on artificial intelligence. The algorithm was tested with 72 random subjects, which covered 11 attributes such as age, gender, height, weight, systolic and diastolic blood pressure, cholesterol, glucose, smoking, alcohol intake, and physical activities. According to the results, the prediction accuracy of the system was $81.62 \%$ at 0.879 precision.
\end{abstract}

Keywords: Cardiovascular diseases, Coronary Artery Disease, Disease Prediction, Machine Learning, Smart Medicine

\section{Introduction}

Cardiovascular disease (CVD) or Cardiac disease is identified as a significant cause of death in both developing and developed countries (Amani and Sharifi 2012). CVD is a class of diseases of the heart or the blood vessels, which includes disorders of the Coronary Artery Disease (CADs), such as angina and myocardial infarction (Murray 2014). There are many associations with CVDs; many of them are related to the atherosclerosis process. Also, CVDs include heart attack, cardiac disease, rheumatic heart disease, cardiomyopathy, irregular heart rhythms, congenital heart defect, and dysfunction of the heart valve, carditis, aortic aneurysms, peripheral artery disease, thromboembolic disease, and venous thrombosis (Lanzkowsky et al. 2016). Atherosclerosis is known as a condition 
that develops in the human body when fat and cholesterol plaque builds up in the walls of the arteries.

According to the World Health Organization (WHO), CVDs are the number one cause of death globally. Total global deaths due to heart diseases were recorded as $31 \%$ by the end of 2020 (www.who.int 2020). Institute for Health Metrics and Evaluation (IHME) of the United States of America (USA) published the Global Burden of Disease Study in 2017. According to the IHME study, deaths caused by cardiovascular diseases since 1990 to 2017 have increased by 5.85 million. Figure 1 illustrates the global death cases due to CVDs since 1990 . Also, $80 \%$ of deaths occur due to stroke and heart attack (Hazra et al. 2017).

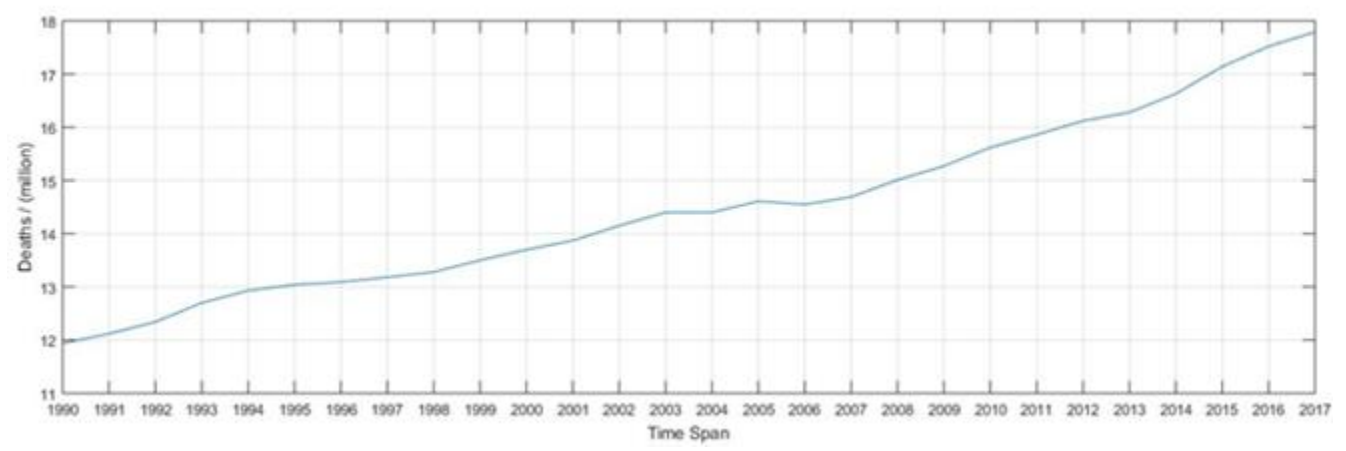

Fig. 1. Deaths - Cardiovascular diseases since 1990 to 2017.

Table 1 depicts the latest WHO data, which was published in 2018 for death in Sri Lanka due to coronary heart disease. According to the data, $22.64 \%$ of deaths were recorded due to the CADs and rank to 94th place among the other countries.

Table 1. Coronary heart disease deaths in Sri Lanka

\begin{tabular}{|l|l|l|l|}
\hline Deaths & Percentage \% & Death Rate & World Rank \\
\hline 28,777 & 22.64 & 123.79 & 94 \\
\hline
\end{tabular}

The most common cause of CVD morbidity and mortality is Ischaemic Heart Diseases (IHD). In 2005, 9.3\% of IHD prevalence was found within Sri Lanka by age and sex (Jayawardene et al. 2017). Cardiovascular diseases or heart diseases mainly refer to Coronary Heart Disease (CHD), Rheumatic Heart Disease (RHD) and Cardiomyopathy (Lopez et al. 2020). Hypertension, Physical inactivity, Smoking, High LDL or Low HDL cholesterol levels and High Blood Pressure are common risk factors of CVDs (Shaima et al. 2016).

The machine learning technique is a modern method to predict cardiovascular diseases that helps to diagnose the patient's health and prevent deaths. Years of 
medical data collection have created a new opportunity for physicians to diagnose patients (Escamila et al. 2020). Nowadays, Machine learning plays an important role in the healthcare industry. Analysis of medical history, symptoms and physical state related to heart disease are the major techniques that are used to predict the diagnosis of a patient. These techniques are followed by medical experts and may cause imprecise diagnosis and often delay due to human error (Singaraju et al. 2011; Haq et al. 2018). Traditional statistical methods, doctor's experiences and medical intuitions are well known key factors that are used to predict the diagnosis (Uddin et al. 2019). Supervised learning algorithms are widely accepted for the training data (Herath et al. 2021; Herath 2021), which involves cardiovascular diseases (Jamgade and Zade 2019). Identification of risk factors is an important part of cardiovascular surveillance (Shah 2011). Risk factors are identified by a brief history of the patient which combined with physical examinations and biochemical tests. There are several behavioral risk factors such as physical inactivity, unhealthy diets, tobacco and alcohol usage, that are responsible for $80 \%$ of Coronary Heart Disease (CHD) (Mohan et al. 2007). The next section of this article examines relevant papers about cardiovascular diseases and their impact in the field of artificial intelligence.

\section{Related Works}

Since Cardiovascular diseases are a global problem many researchers are studying different techniques to diagnoses and predict CVDs. In this section, we discussed several research studies related to CVDs.

Y. Chauhan from the Parul University of India have published an article about classification algorithms of machine learning related to cardiovascular disease prediction in 2018. He used a logistic regression dataset from an on-going cardiovascular study of Framingham and Massachusetts from Kaggle (Chauhan 2020). According to Chauhan, logistic regression is the most suitable algorithm for disease prediction. Further, his research shows the logistic regression model was $89 \%$ more accurate than the K-Nearest Neighbours (KNN), Support Vector Machine (SVM), Decision Tree and Random Forest.

Previous studies led by Chaithra and Madhu at Jagadguru Sri Shivarathreeshwara University have followed a study to analyse the various data mining techniques for predicting cardiovascular disease (Chaithra and Madhu 2018). They 
investigate three different classification models to perform this study. The analysis showed that the prediction results of the neural network was $97.91 \%$ more accurate than the J48 Decision Tree and Naïve Bayes.

Sahid and his research team from the University of Management and Technology of Pakistan have enlightened several techniques in artificial neural networks (Awan et al. 2018). According to them, 94.7\% accuracy observed by predicting heart disease using an artificial neural network method.

Rastogi, Chaturvedi, Satya and Arora have performed a research on development of a heart disease prediction based on the physical and mental parameters of the patients (Rastogi et al. 2020). They used features such as sex, cholesterol level, blood pressure, Tension-Type Headache (TTH) and stress level to predict heart diseases. They have also used different types of data mining methods to analyse the heart disease dataset. Support vector machine (SVM), Naive Bayes and Decision Trees were the major data mining techniques which they used to compare the results.

Higuchi, Sato, Makuuchi, Furuse, Takamoto and Takeda have tested and verified results for three layered artificial neural networks to predict the condition of the patient's heart. They used ANNs for analysing the phonocardiogram data to diagnose the patients. According to Higuchi and his team, the accuracy of the diagnoses can be improved by future data accumulation (Higuchi et al. 2006). Maciej, Piotr, Jacek, Joseph, Jay and Georgia from the University of Louisville in USA have investigated the class imbalance due to the training of the dataset while developing the neural network system for the medical diagnosing computerized systems. According to their results, Backpropagation was more performable compared to the Particle Swarm Optimization (PSO) for imbalanced training data. They also mentioned that this imbalance occurred due to the small data sample and the large number of features associated with the dataset (Mazurowski et al 2008).

The next section of this article describes the design methodology of the proposed algorithm.

\section{Methodology}

The ultimate goal of this research is to predict coronary artery disease using the artificial neural network-based approach. Cardiovascular disease dataset has 
70,000 observations from medical examinations. The methodology of this study was comprised of five main steps,

1. Find the risk factors to predict cardiovascular disease.

2. Development of an Artificial Neural Network (ANN) Model.

3. Select a suitable transfer function for the node activation.

4. Training an artificial neural network using the back propagation Levenberg Marquardt (LM) algorithm.

5. Perform the test run and analyses results using the data analysing method.

Figure 2 illustrates the design model of the proposed algorithm.

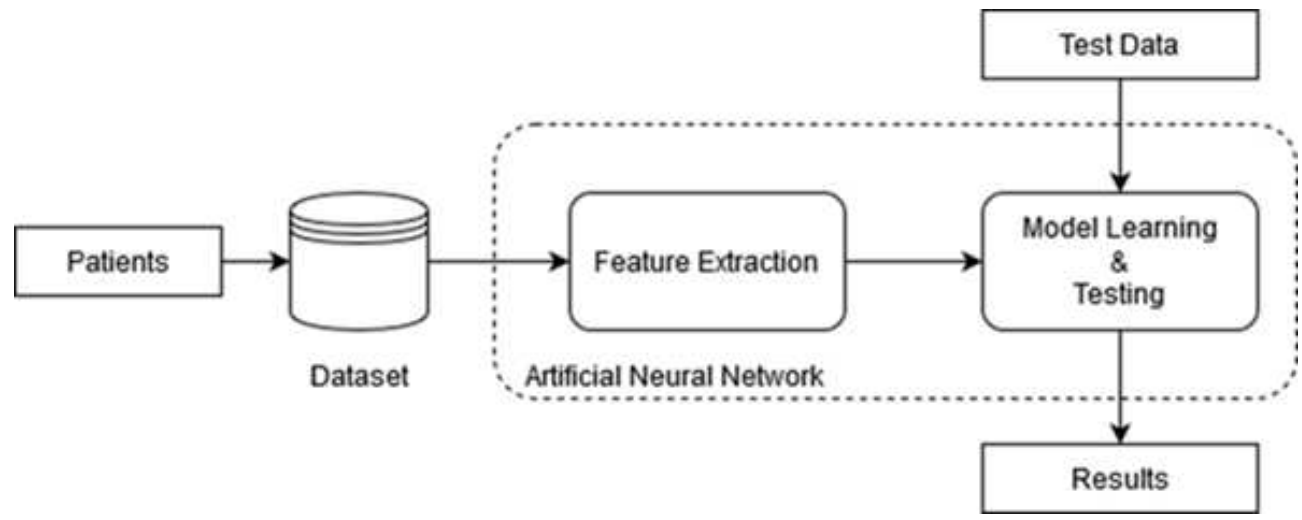

Fig. 2. Design model of the proposed algorithm

\section{Description of Dataset}

The "Kaggle cardiovascular dataset" is widely used by the researchers for predicting cardiac disease (Ulianova 2019). The dataset consists of 70,000 records in which information was given by the patients at the moment of medical examination. The dataset contains 12 features that include four objective features, four examination features, three subjective features, and one target variable for the presence or absence of cardiovascular disease. Table 2 depicts the features extracted from the dataset. Table 3 demonstrates the output features of the dataset.

Table 2. Input features of the dataset

\begin{tabular}{|l|l|}
\hline Input Features & Parameter \\
\hline Objective & $\begin{array}{l}\text { Age (days), Height (cm), Weight (kg), } \\
\text { Gender (Male, Female) }\end{array}$ \\
\hline Examination & $\begin{array}{l}\text { Systolic blood pressure (mmHg), } \\
\text { Diastolic blood pressure (mmHg), } \\
\text { Cholesterol (Normal, Above normal, } \\
\text { Well above normal), Glucose (Normal, } \\
\text { Above normal, Well above normal) }\end{array}$ \\
\hline Subjective & Smoking (Yes, No), Alcohol intake \\
\hline
\end{tabular}


Table 3. Output feature in of the dataset

\begin{tabular}{|l|l|}
\hline Output Feature & Parameter \\
\hline Target Variable & Cardiovascular disease (Percentage) \\
\hline
\end{tabular}

\section{Development of an Artificial Neural Network Algorithm}

Due to our study associated with the multilayer perceptron type, the procedure of the teaching algorithm for MLP was performed as follows,

- The structure of the neural network was defined by initializing the activation function, network parameters, weights, and biases. The transfer function was used to convert an input to the output signal of the model. This study was referred to as the hyperbolic tangent transfer function and Tansig function was used to activate the function of the network. This hyperbolic tangent transfer function was related to the sigmoid function, which gave output between -1 to 1 .

- The parameters associated with the training algorithm were defined. The algorithm was developed with the random data division and Levenberg Marquardt training algorithm. The performance was measured using the Mean Square Error (MSE) technique. The training algorithm was performed with 1000 iterations during the approximately eight minutes of time. The performance of the training algorithm was recorded as 0.178 rates and the gradient of the learning algorithm provides that steepest direction. The goals of the machine learning techniques are to reach an optimal solution, which is also known as the bottom of the bowl to give the direction of the maximum rate change. In this study, the gradient was recorded as 0.000322 within the range between 0.643 and $1.00 \times 10-7$.

- After the neural network developed, 72 test subjects were observed and verified accuracy with real values.

Figure 3 illustrates the proposed Multilayer Perception Model (MLP) of the Artificial Neural Network (ANN) with eleven attributes in input layer, fifteen nodes in first hidden layer, five nodes in second hidden layer and one node in output layer.

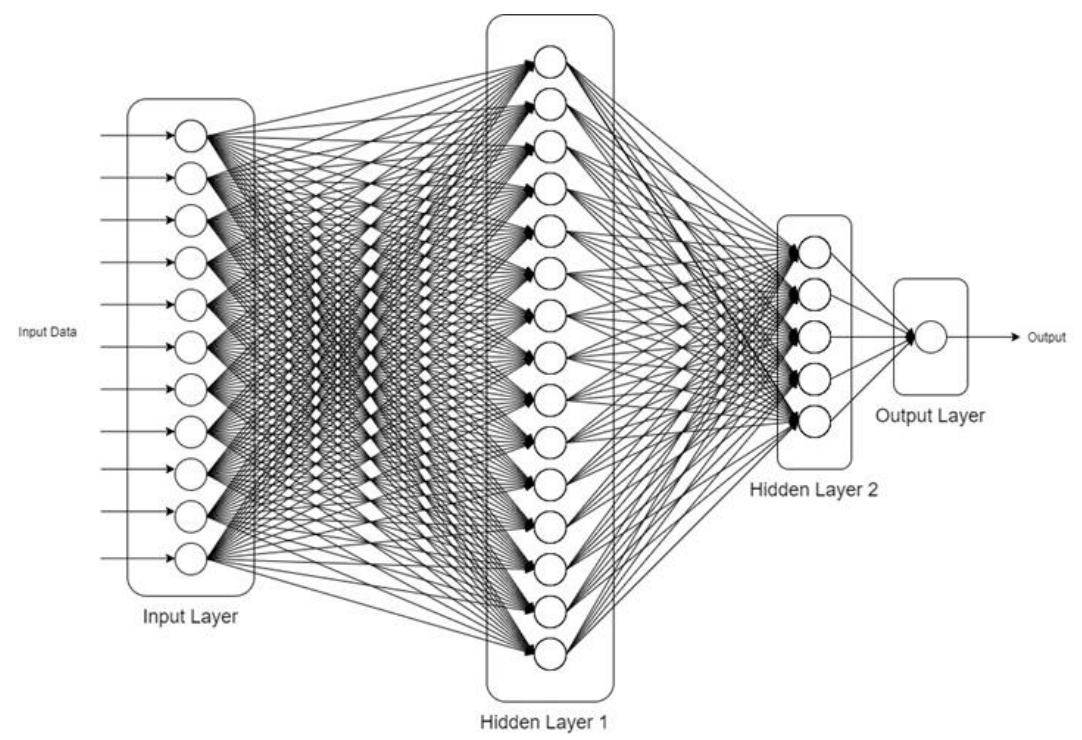

Fig. 3. Architecture of the proposed artificial neural network model. 
The hyperbolic tangent sigmoid transfer function is widely used as the activation function for the hidden layer of a neural network. The activation function is a mathematical representation that helps to determine the output of a neural network. The activation function is associated with each neuron and determines the state of activation, whether the neuron should fire or not. This activation status is based on the neuron's input state relevant to the prediction of the model. The Sshaped anti-symmetric function was used for input to output transformation (Freitas et al. 2018). This transformation allows the output of each neuron to stay between -1 and +1 in the interval. The hyperbolic tangent sigmoid function is defined by the eq. (1),

$$
a=\operatorname{Tansig}(n)=\frac{2}{1+e^{-2 n}}-1
$$

\section{Back Propagation Training Method}

Backpropagation is an algorithm in supervised learning to train multi-layer perceptron. It is the method by which the weights of a neural net are fine-tuned based on the error rate obtained in the previous epoch. Levenberg Marquardt (LM) algorithm was used in this study to perform backpropagation training. LM algorithm is a hybrid algorithm that is a combination of the Gauss-Newton method and the Gradient Descent method. The sum of square error is defined in the Eq. (2),

$$
E=\frac{1}{2} \sum k\left(e_{k}\right)^{2}=\frac{1}{2}\|e\|^{2}
$$

Where, $e_{k}$ is the error in the kth example, $e$ is a vector with elements $e_{k}$. The error vector is defined by Taylor series as per Eq. (3),

$$
e_{(j+i)}=e_{(j)}+\frac{\partial e_{k}}{\partial w_{i}}\left(w_{(j+1)}-w_{(j)}\right)
$$

The error function is express as follows in the Eq. (4),

$$
E=\frac{1}{2}\left\|e_{(j)}+\frac{\partial e_{k}}{\partial w_{i}}\left(w_{(j+1)}-w_{(j)}\right)\right\|^{2}
$$

Minimizing the error function that respect to the new weights and vectors describes in the Eq. (5),

$$
w_{(j+1)}=w_{(j)}-\left(Z^{T} Z\right)^{-1} Z^{T} e_{(j)}
$$


Where, $(z)_{k i}=\frac{\partial e_{k}}{\partial w_{i}}$

Since the Hessian for the sum of the square error function is described as follows from the Eq.(6),

$$
(H)_{i j}=\frac{\partial^{2} E}{\partial w_{i} \partial w_{j}}=\sum\left\{\left(\frac{\partial e_{k}}{\partial w_{i}}\right)\left(\frac{\partial e_{k}}{\partial w_{i}}\right)+e_{k} \frac{\partial^{2} e_{k}}{\partial w_{i} \partial w_{j}}\right\}
$$

By neglecting the second term, we can write $H=Z^{T} Z$

Updating of the weights is involved with the inverse Hessian or an approximation theory of the nonlinear networks. Since Hessian is a first-order derivative equation, the computations are not complex and respected to the network weights. The updating formula is applied iteratively to minimize the error function, which results in a large step size.

In the Levenberg Marquardt algorithm, the error function needs to be minimized while keeping the small step size. This phenomenon ensures the validity of the linear approximation and is accomplished by aiding in modifying the error function (Sapna et al. 2012) as on the Eq. (7).

$$
E=\frac{1}{2}\left\|e_{(j)}+\frac{\partial e_{k}}{\partial w_{i}}\left(w_{(j+1)}-w_{(j)}\right)\right\|^{2}+\lambda\left\|w_{(j+1)}-w_{(j)}\right\|^{2}
$$

Where $\lambda$ is the parameter for governing the step size. Minimizing the modified error with respect to $w_{(\mathrm{j}+1)}$ is described as in the Eq. (8),

$$
w_{(j+1)}=w_{(j)}-\left(Z^{T} Z+\lambda I\right)^{-1} Z^{T} e_{(j)}
$$

For a very large value of $\lambda$ amount, the standard gradient descent method is used. For very small values of $\lambda$ amount, the newton method is used.

\section{Results and Analysis}

A total of 72 test subjects were obtained from the Kaggle Cardiovascular disease dataset with 11 attributes. The entire dataset was classified into four classes; each class composed of 18 test subjects and used to validate the results.

The progress is constantly being updated during the training. The performance magnitude of the performance, gradient, and the number of validation checks are of most interest during the training process. To end the instruction, the magnitude 
of the gradient and the amount of validity tests are used. When the preparation approaches a minimum output, the gradient can become very low. Figure 4 illustrates the Regression graph of the Training.

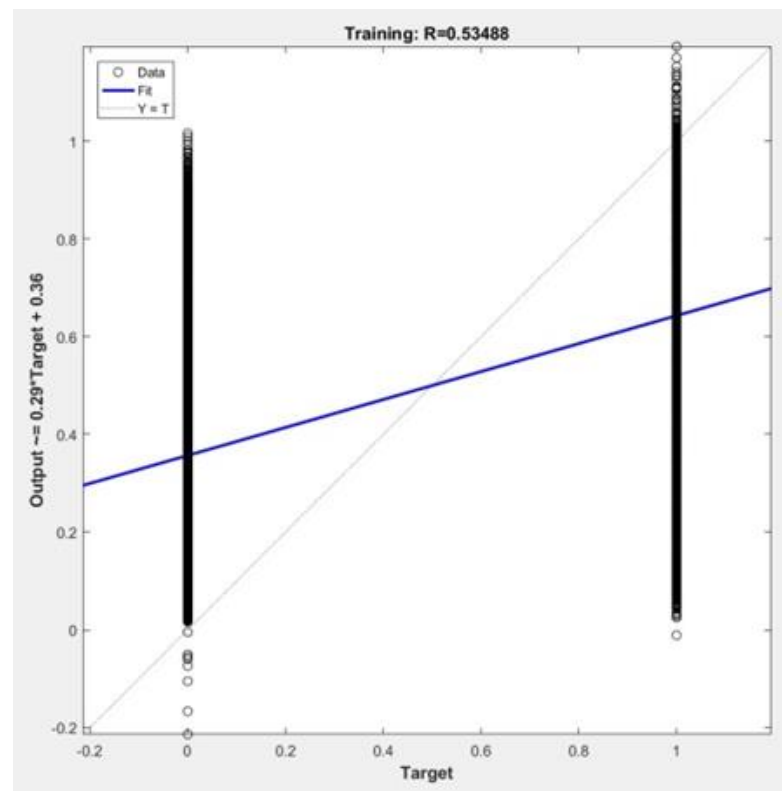

Fig. 4. Regression graph of the training.

If the gradient magnitude is less than $1 \times 10-7$, otherwise, the preparation must cease. We were adjusted the limit by setting the net.trainParam.min grad parameter. The number of validation checks reflects the number of successive iterations that fail to reduce the efficiency of the validation. The performance plot displays the performance function value versus the number of iterations. It constructs training, validation, and performance tests. The training state plot shows the progress of other training variables, such as gradient magnitude, the number of validation inspections, etc. The plot for the histogram error shows the distribution of network errors.

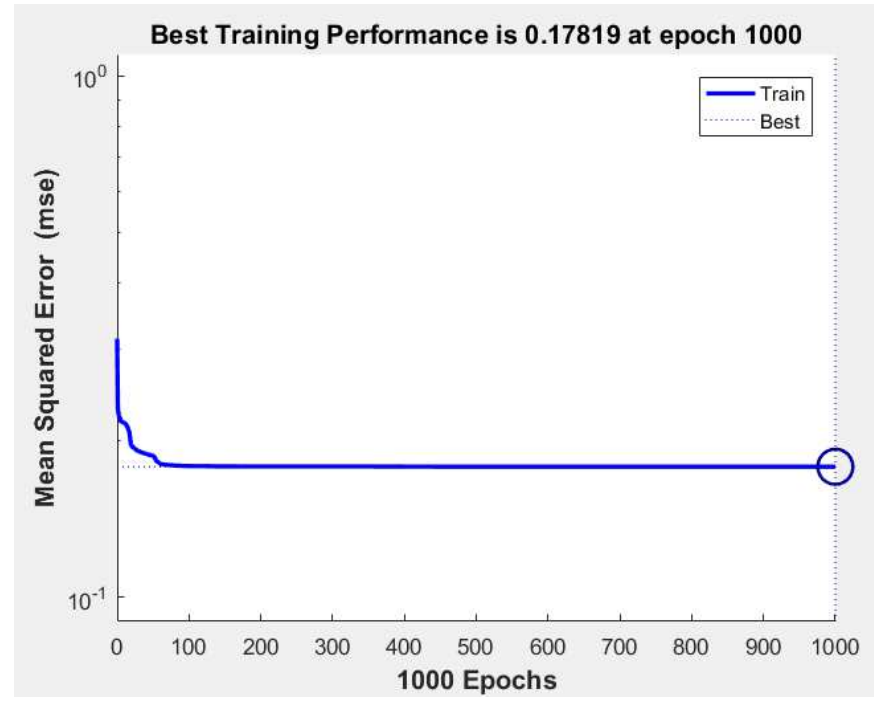

Fig. 5. Mean square error vs. training performance. 
Upon training and testing of the network, the network object may be used to measure the response to any input data. Figure 5 illustrates the Mean Square Error verses training performance of the neural network.

To grasp the actions of the classifier, we were calculated the confusion matrix metric. A confusion matrix, also known as an error matrix, is widely used in the area of machine learning and especially the issue of statistical classification. A confusion matrix is a table often used to describe a performance of a classification model or classifier on a set of test data for which the true values are known.

Confusion Matrix validates the performance of an algorithm.

Table 4. Confusion matrix.

\begin{tabular}{|c|c|c|c|}
\hline & \multicolumn{2}{|c|}{ Predicted class } \\
\hline & & Positive & Negative \\
\hline \multirow{2}{*}{$\frac{\frac{\tilde{V}}{\tilde{E}}}{\frac{\tilde{U}}{\tilde{U}}}$} & Positive & $\begin{array}{c}\text { True } \\
\text { Positive }\end{array}$ & $\begin{array}{c}\text { False } \\
\text { Negative }\end{array}$ \\
\hline & Negative & $\begin{array}{c}\text { False } \\
\text { Positive }\end{array}$ & $\begin{array}{c}\text { True } \\
\text { Negative }\end{array}$ \\
\hline
\end{tabular}

Table 4 displays the confusion matrix for a question of classification between two groups. It is a table of contingency that includes details regarding current and expected classifications provided by a method of classification. This is a review of findings from forecasts on a classified problem. The number of observations that are right and inaccurate was listed with count values and broken down by category.

The entries in the confusion metrics were calculated from the coincidence matrix by using the following hypothesis,

- True Negative (TN) is the number of correct predictions that an instance is negative.

- True Positive (TP) is the number of correct predictions that an instance is positive.

- False Positive (FP) is the number of incorrect predictions that an instance is positive.

- False Negative (FN) is the number of incorrect predictions that an instance is negative. The accuracy was calculated by using the Eq. (9),

$$
\text { Accuracy }=\frac{T P+T N}{T P+T N+F P+F N}
$$

Classification Matrix shows the frequency of correct and faulty predictions (Kim et al. 2015). It compares the actual test data set values to the predicted values in the trained model. Table 5 depicts the Classification Matrix results for all possibilities; $86 \%$ of patients are correctly diagnosed as having or not having the 
disease and predicted as having it or not. $14 \%$ of patients are misdiagnosed as they don't have the disease, but they had it.

Table 5. Results of confusion matrix for the algorithm.

\begin{tabular}{|l|l|c|c|}
\hline Class & \multirow{2}{*}{ Actual } & \multicolumn{2}{|l|}{ Predicted } \\
\cline { 3 - 4 } & & Positive & Negative \\
\hline $\mathbf{1}$ & Positive & 7 & 1 \\
\cline { 2 - 4 } & Negative & 2 & 8 \\
\hline $\mathbf{2}$ & Positive & 6 & 1 \\
\cline { 2 - 4 } & Negative & 1 & 10 \\
\hline $\mathbf{3}$ & Positive & 8 & 2 \\
\cline { 2 - 4 } & Negative & 0 & 8 \\
\hline $\mathbf{4}$ & Positive & 8 & 1 \\
\cline { 2 - 4 } & Negative & 1 & 8 \\
\hline
\end{tabular}

Mean Absolute Error (MAE) is used to measure the error between pairs of observations in statistics, and MAE is a function that is an evaluation metric used by regression simulations (Sammut and Webb 2011). A model's mean absolute error about a test set is the sum of the absolute values of the actual estimation errors in the test set for all instances. The verified indicator Mean Absolute Error (MAE) was calculated using the Eq. (10):

$$
\mathrm{MAE}=\frac{\sum_{t=1}^{N}\left|Y_{t}-F_{t}\right|}{N}
$$

Where $Y_{t}$ is the real data (verification); $F_{t}$ is the prediction data.

The smaller the MAE, the closer the prediction data is to the real data (verification), and the larger the $M A E$, the greater the difference between the predicted data and the real data (verification). The experimental findings revealed that neural network was outperforming in the area of forecasting cases of cardiovascular disease. The experiment was designed to determine Neural Network output and explore the impact of the selection of attributes on the model.

Table 6. Accuracy and MAE of each class.

\begin{tabular}{|c|c|c|c|c|}
\hline 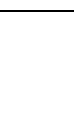 & Subject Class & $\begin{array}{l}\text { Mean Absolute } \\
\text { Error (MAE) }\end{array}$ & Precision & $\begin{array}{l}\text { Accuracy } \\
(\%)\end{array}$ \\
\hline 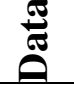 & ही 1 & 0.2003 & 0.7777 & 79.97 \\
\hline
\end{tabular}




\begin{tabular}{|l|l|l|l|l|}
\hline 2 & 0.1576 & 0.8571 & 84.24 \\
\hline 3 & 0.1752 & 1.0000 & 82.48 \\
\hline 4 & 0.2021 & 0.8888 & 79.79 \\
\cline { 2 - 5 } & \multicolumn{2}{|l}{ Overall accuracy and precision } & 0.8788 & 81.62 \\
\hline
\end{tabular}

Figure 6 to Figure 9 show the test result variation of classes 1 to 4 . Mean absolute errors respectively in class 1 to 4 are $0.2003,0.1576,0.1752$ and 0.2021 .

This test dataset contains a total of 72 subjects in four classes. Neural Network has proved its performance in terms of accuracy (81.62\%), which makes it an excellent classifier to be used in the medical field for classification and prediction. Table 6 illustrates the accuracy and mean absolute error of each class.

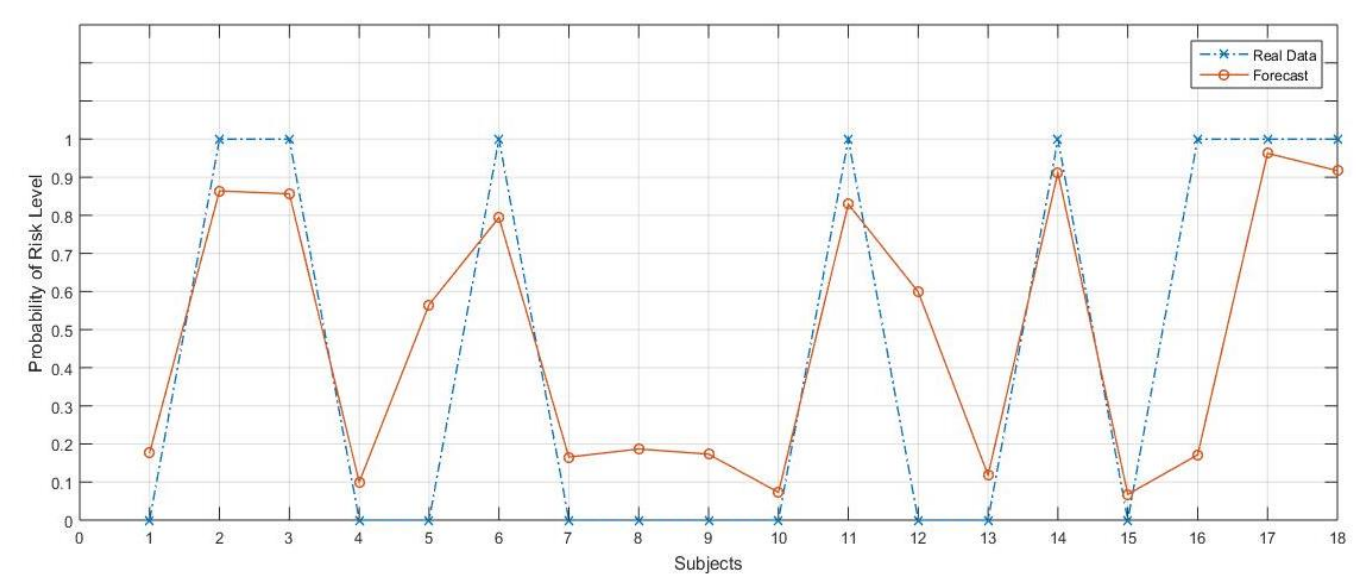

Fig. 6. Test subjects vs. probability of risk level (Class 1).

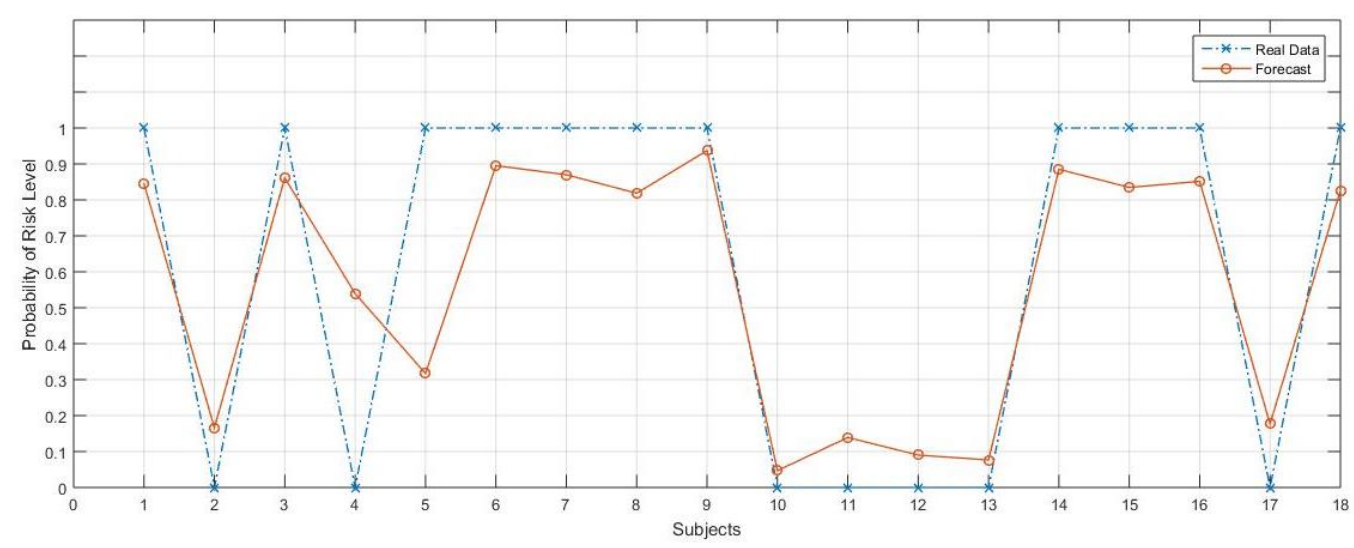

Fig. 7. Test subjects vs. probability of risk level (Class 2). 


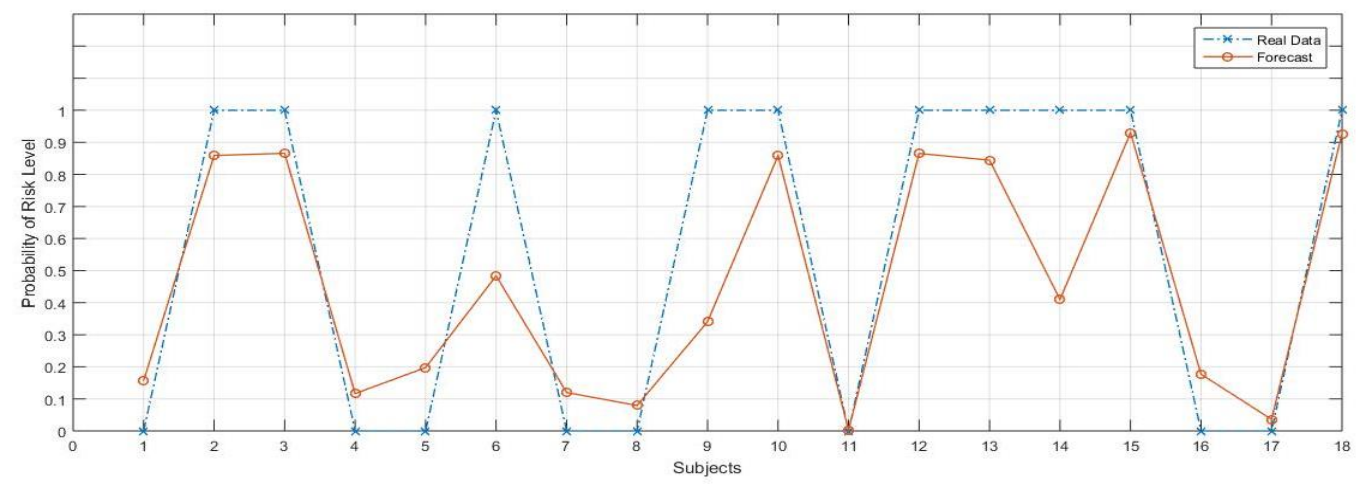

Fig. 8. Test subjects vs. probability of risk level (Class 3).

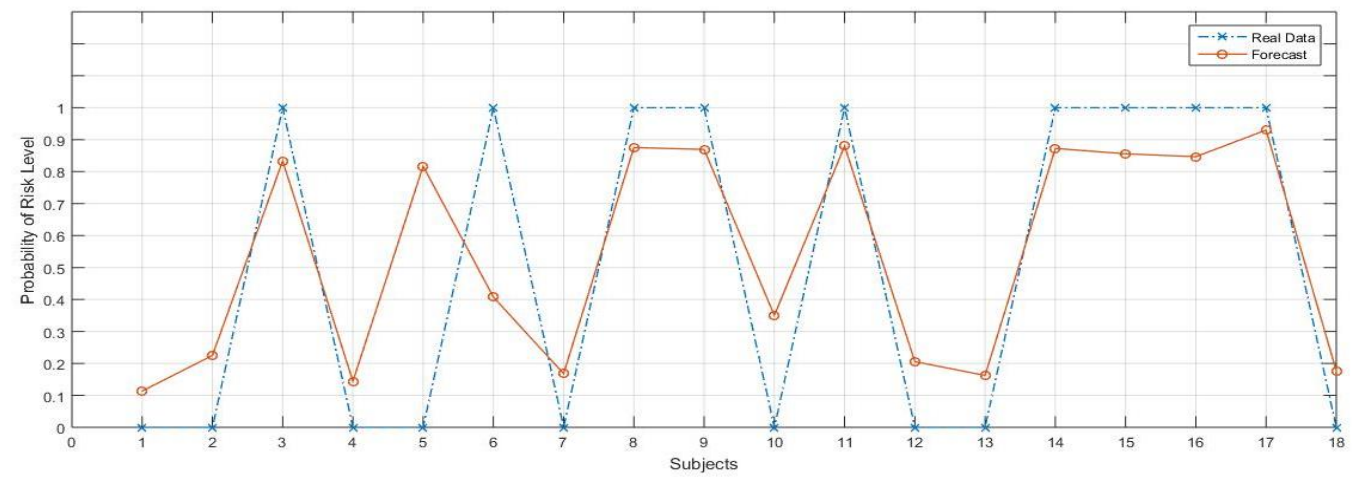

Fig. 9. Test subjects vs. probability of risk level (Class 4).

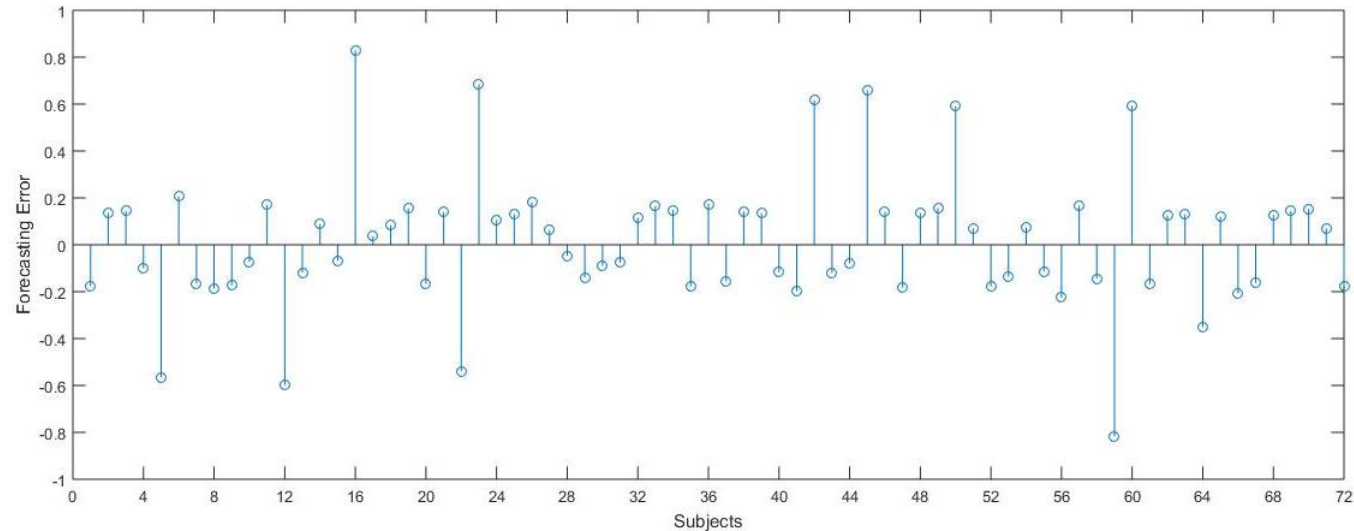

Fig. 10. Forecasting error between real and predicted data 


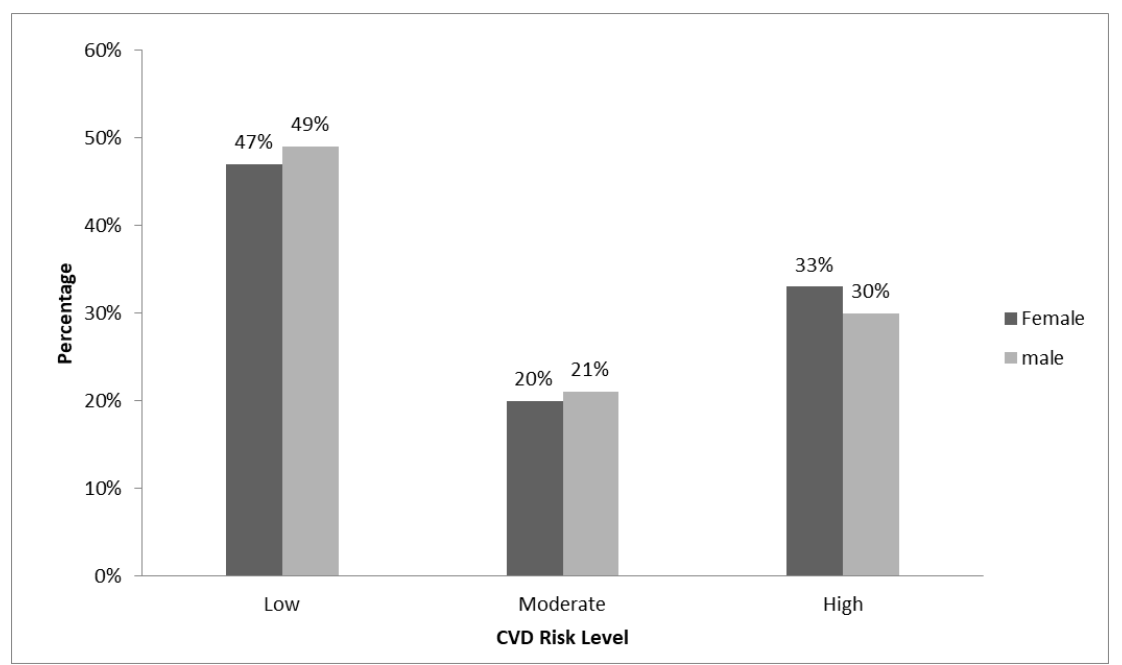

Fig. 21. Variation of the CVD risk level.

Figure 10 indicates the difference between the real data and the predicted results for the method forecasting Accuracy. The Mean Absolute Error (MAE) is 0.1832. The decomposed data can reflect fluctuation information on different subjects while retaining the characteristics of the original data. Figure 11 shows the degree of CVD danger, and $48.0 \%$ of topics were at low CVD risk points for both males and females. Average risk forecasting for moderate and high risk for both genders was $20.5 \%$ and $31.5 \%$ respectively.

\section{Conclusions}

In this article, we proposed a possible solution for predicting cardiovascular diseases using artificial intelligence technology. Multilayer perceptron neural networks, backpropagation (Levenberg Marquardt) training algorithm, and tansig activation function was used to develop the prediction system. The excremental results suggested that the system predict presence of cardiovascular disease with $81.62 \%$ efficiency. The accuracy of the results may increase by engaging with more attributes such as chest pain type, heredity, stress level and treatment for hypertension, etc.

\section{Acknowledgment}

The authors want to express their gratitude to Dr. U.D.C. Nimanthi, a physician at the National Eye Hospital of Sri Lanka, Colombo 10 - Sri Lanka, for reviewing medical background of this article. 


\section{Conflicts of Interest}

The authors are declaring that they have no conflicts of interest.

\section{References}

Awan, S. M., Riaz, M. U., \& Khan, A. G. (2018). Prediction of heart disease using artificial neural network. VFAST Transactions on Software Engineering, 6(1), 51-61.

Chaithra, N., \& Madhu, B. (2018). Classification models on cardiovascular disease prediction using data mining techniques. Journal of Cardiovascular Diseases and Diagnosis.

Chauhan, Y. J. Cardiovascular Disease Prediction using Classification Algorithms of Machine Learning.

Freitas, D., Lopes, L. G., \& Morgado-Dias, F. (2018, July). A neural network based approach for approximating real roots of polynomials. In Proceedings of the International Conference on Mathematical Applications (ICMA), Funchal, Portugal (pp. 9-12).

Gárate-Escamila, A. K., El Hassani, A. H., \& Andrès, E. (2020). Classification models for heart disease prediction using feature selection and PCA. Informatics in Medicine Unlocked, 19, 100330.

Haq, A. U., Li, J. P., Memon, M. H., Nazir, S., \& Sun, R. (2018). A hybrid intelligent system framework for the prediction of heart disease using machine learning algorithms. Mobile Information Systems, 2018.

Hazra, A., Mandal, S. K., Gupta, A., Mukherjee, A., \& Mukherjee, A. (2017). Heart disease diagnosis and prediction using machine learning and data mining techniques: a review. Advances in Computational Sciences and Technology, 10(7), 2137-2159.

Herath, H. M. K. K. M. B. (2021). Internet of Things (IoT) Enable Designs for Identify and Control the COVID-19 Pandemic. In Artificial Intelligence for COVID-19. Springer, Cham. Herath, H. M. K. K. M. B., Karunasena, G. M. K. B., \& Herath, H. M. W. T. (2021). Development of an IoT Based Systems to Mitigate the Impact of COVID-19 Pandemic in Smart Cities. In Machine Intelligence and Data Analytics for Sustainable Future Smart Cities (pp. 287-309). Springer, Cham.

Higuchi, K., Sato, K., Makuuchi, H., Furuse, A., Takamoto, S., \& Takeda, H. (2006). Automated diagnosis of heart disease in patients with heart murmurs: application of a neural network technique. Journal of medical engineering \& technology, 30(2), 61-68.

Jamgade, A. C., \& Zade, S. D. (2019). Disease prediction using machine learning. International Research Journal of Engineering and Technology, 6(5), 6937-6938.

Jayawardene, J. B., Samarutilake, G. D. N., Zackie, M. H. M., De Silva, V., Karunanayake, A., \& Weerasooriya, M. A. (2017). Prevalence of coronary artery disease in a semi urban population in Southern Sri Lanka. Ceylon Medical Journal, 62(1).

Vanisree, K., \& Singaraju, J. (2011). Decision support system for congenital heart disease diagnosis based on signs and symptoms using neural networks. International Journal of Computer Applications, 19(6), 6-12. 
Kim, J., Lee, J., \& Lee, Y. (2015). Data-mining-based coronary heart disease risk prediction model using fuzzy logic and decision tree. Healthcare informatics research, 21(3), 167.

Lanzkowsky, P., Lipton, J. M., \& Fish, J. D. (Eds.). (2016). Lanzkowsky's manual of pediatric hematology and oncology. academic press.

Lopez, E. O., Ballard, B. D., \& Jan, A. (2020). Cardiovascular disease. StatPearls [Internet]. Mazurowski, M. A., Habas, P. A., Zurada, J. M., Lo, J. Y., Baker, J. A., \& Tourassi, G. D. (2008). Training neural network classifiers for medical decision making: The effects of imbalanced datasets on classification performance. Neural networks, 21(2-3), 427-436.

Mohan, V., Sandeep, S., Deepa, R., Shah, B., \& Varghese, C. (2007). Epidemiology of type 2 diabetes: Indian scenario. The Indian journal of medical research, 125(3), 217-30.

Murray,C.J.L..(2014). Global, regional, and national age-sex specific all-cause and cause-specific mortality for 240 causes of death, 1990-2013: a systematic analysis for the Global Burden of Disease Study 2013. The Lancet, 385(9963), 117-171.

Rastogi, R., Chaturvedi, D. K., Satya, S., \& Arora, N. (2020). Intelligent heart disease prediction on physical and mental parameters: a ML based IoT and big data application and analysis.

Machine Learning with Health Care Perspective. Learning and Analytics in Intelligent Systems, 13, 199-236.

Sammut, C., \& Webb, G. I. (Eds.). (2011). Encyclopedia of machine learning. Springer Science \& Business Media.

Sapna, S., Tamilarasi, A., \& Kumar, M. P. (2012). Backpropagation learning algorithm based on Levenberg Marquardt Algorithm. Comp Sci Inform Technol (CS and IT), 2, 393-398.

Shah, S. (2011). Primary prevention of cardiovascular disease. InnovAiT.

Shaima, C., Moorthi, P. V., \& Shaheen, N. K. (2016). Cardiovascular diseases: Traditional and non-traditional risk factors. Journal of Medical \& Allied Sciences, 6(2), 46.

Uddin, S., Khan, A., Hossain, M. E., \& Moni, M. A. (2019). Comparing different supervised machine learning algorithms for disease prediction. BMC medical informatics and decision making, 19(1), 1-16.

Ulianova, S. (2019). Cardiovascular Disease dataset. Kaggle. Retrieved June 27, 2020, from https://www.kaggle.com/sulianova/cardiovascular-disease-dataset.

Amani, R., \& Sharifi, N. (2012). Cardiovascular disease risk factors. The cardiovascular systemphysiology, diagnostics and clinical implications. InTech, Rijeka, 279-310.

World Health Organization. Cardiovascular diseases (CVDs). Retrieved June 27, 2020, from https://www.who.int/en/news-room/fact-sheets/detail/cardiovascular-diseases-(cvds). 